\title{
UNIFORM TREATMENT OF DARBOUX'S METHOD AND THE HEISENBERG POLYNOMIALS
}

\author{
SAI-YU LIU, R. WONG, AND YU-QIU ZHAO \\ (Communicated by Walter Van Assche)
}

Abstract. We show that the set of Heisenberg polynomials furnishes a simple non-trivial example in the uniform treatment of Darboux's method.

\section{The Heisenberg polynomials}

The Heisenberg polynomials are polynomials in $z$ and $\bar{z}$, defined explicitly as

$$
C_{n}^{(\alpha, \beta)}(z)=\sum_{j=0}^{n} \frac{(\alpha)_{j}(\beta)_{n-j}}{j !(n-j) !} \bar{z}^{j} z^{n-j}, n=0,1, \cdots,
$$

where $\alpha$ and $\beta$ are real constants, and $(\gamma)_{k}$ is the Pochhammer symbol defined by $(\gamma)_{0}=1$ and $(\gamma)_{k}=\gamma(\gamma+1) \cdots(\gamma+k-1)$. The representation can be readily derived from the generating function

$$
(1-w \bar{z})^{-\alpha}(1-w z)^{-\beta}=\sum_{n=0}^{\infty} C_{n}^{(\alpha, \beta)}(z) w^{n}, \quad|w z|<1 .
$$

The notation $C_{n}^{(\alpha, \beta)}(z)$ was used by Gasper in [8] in the sense of (1.1) and (1.2), and yet the term Heisenberg polynomials was first used by Dunkl in [2].

The study of Heisenberg polynomials may be traced back to the investigation of the harmonic polynomials on Heisenberg group $H_{N}$. The group $H_{N}$ consists of $(N+1)$-tuples in $\mathbb{C}^{N} \times \mathbb{R}, N=1,2,3 \cdots$, with the group operation

$$
(\zeta, s) \cdot(z, t)=(\zeta+z, s+t+2 \operatorname{Im}\langle\zeta, z\rangle),
$$

where $\zeta, z \in \mathbb{C}^{N}, s, t \in \mathbb{R}$, and $\langle\zeta, \xi\rangle=\sum_{j=1}^{N} \zeta_{j} \bar{\xi}_{j}$; cf. Dunkl [2] and Greiner [9].

The Heisenberg group $H_{N}$ arose in various branches of mathematics, such as harmonic analysis [10, 16] and partial differential operators and equations [6, 7]. The reason may partially be that the results in Abelian harmonic analysis could be adapted to Heisenberg groups.

The so-called $L_{2 \gamma}$-harmonic polynomials are the polynomial solutions $p=$ $p\left(z_{1}, \bar{z}_{1}, \cdots z_{N}, \bar{z}_{N}, t\right)$ of $L_{2 \gamma} p=0$ in $H_{N}$, where the differential operator $L_{2 \gamma}$ is

Received by the editors August 18, 2011 and, in revised form, October 22, 2011.

2010 Mathematics Subject Classification. Primary 41A60; Secondary 33C15.

Key words and phrases. Heisenberg polynomials, Darboux's method, uniform asymptotic expansion, confluent hypergeometric function, Bessel function.

The research of the third author was supported in part by the National Natural Science Foundation of China under grant numbers 10471154 and 10871212. 
defined by

$$
L_{2 \gamma}=-\frac{1}{2} \sum_{j=1}^{N}\left(Z_{j} \bar{Z}_{j}-\bar{Z}_{j} Z_{j}\right)+2 i \gamma \frac{\partial}{\partial t}
$$

and

$$
Z_{j}=\frac{\partial}{\partial z_{j}}+i \bar{z}_{j} \frac{\partial}{\partial t}, \bar{Z}_{j}=\frac{\partial}{\partial \bar{z}_{j}}-i \bar{z}_{j} \frac{\partial}{\partial t},
$$

$\gamma$ being a constant. In particular, $L_{0}$ in $H_{1}$ is a sub-elliptic Laplacian on $\mathbb{R}^{3}$; cf. 6. 7.

Greiner [9] initiated the study of $L_{2 \gamma}$-spherical harmonic polynomials. It is later shown by Dunkl (cf. 2]) that every such polynomial can be expressed in terms of $C_{n}^{(\alpha, \beta)}$.

A few facts are known about the Heisenberg polynomials. For example, the representation (1.1) of $C_{n}^{(\alpha, \beta)}$ can be interpreted as a Gauss hypergeometric function. The polynomials $C_{n}^{(\alpha, \beta)}(z)$ reduce to the Legendre polynomials on the unit circle when $\alpha=\beta=\frac{1}{2}$. The reader is referred to [2] for more properties of these polynomials.

We mention several relevant investigations on the Heisenberg polynomials. In 1981, Gasper [8] indicated that $\left\{C_{n}^{(\alpha, \beta)}\left(e^{i \theta}\right)\right\}$ furnishes a system of orthogonal functions on $[0,2 \pi]$, with weight function $w^{(\alpha, \beta)}(\theta)=\left(1-e^{-2 i \theta}\right)^{\alpha}\left(1-e^{2 i \theta}\right)^{\beta}$, where $\alpha, \beta, \alpha+\beta>-1$.

In 1984, Dunkl 22 obtained a bi-orthogonality relation for these functions in $L^{2}\left(T,|\sin \theta|^{\alpha+\beta} d \theta\right)$, where $\alpha>0, \beta>0$, and $T=\left\{e^{i \theta} \mid-\pi<\theta \leq \pi\right\}$. A 1986 paper [3] of Dunkl addressed the density problem for $\left\{C_{n}^{(\alpha, \beta)}\left(e^{i \theta}\right)\right\}$ in $L_{2}$.

In 1986, Temme [15] considered two sets of bi-orthogonal polynomials, $P_{n}(z ; \alpha, \beta)$ and $Q_{n}(z ; \alpha, \beta)$, closely related to the Heisenberg polynomials, where

$$
P_{n}(z ; \alpha, \beta)=F(-n, \alpha+\beta+1 ; 2 \alpha+1 ; 1-z) \text { and } Q_{n}(z ; \alpha, \beta)=P_{n}(z ; \alpha,-\beta),
$$

with $F(a, b ; c ; z)$ being the Gauss hypergeometric function; cf. [13, p. 384]. The connection with the Heisenberg polynomials is

$$
P_{n}\left(e^{i \theta} ; \alpha, \beta\right)=\frac{n !}{(2 \alpha+1)_{n}} e^{i n \theta / 2} C_{n}^{(\alpha-\beta, \alpha+\beta+1)}\left(e^{i \theta / 2}\right) .
$$

The two sets $\left\{P_{n}\right\}$ and $\left\{Q_{n}\right\}$ are bi-orthogonal on the unit circle with respect to the weight function $\left(1-e^{i \theta}\right)^{\alpha+\beta}\left(1-e^{-i \theta}\right)^{\alpha-\beta}$. Based on an integral representation of the Gauss hypergeometric function, uniform asymptotic expansions for $P_{n}(z ; \alpha, \beta)$ were obtained in [15], including error bounds. These expansions are in terms of confluent hypergeometric functions, and show that all points on the unit circle in the $z$-plane are transition points in the sense that the polynomials have different asymptotic behavior inside and outside the unit disk. To ensure the convergence of the integral representation, extra restrictions on the parameters, namely $\alpha+\beta>-1$ and $\alpha-\beta \geq 0$, are required in $[15$.

It is readily seen that the Heisenberg polynomials are homogeneous functions in $z$ and $\bar{z}$, which implies that $C_{n}^{(\alpha, \beta)}\left(\rho e^{i \theta}\right)=\rho^{n} C_{n}^{(\alpha, \beta)}\left(e^{i \theta}\right)$. Hence, to study the behavior of the polynomials, it is natural to consider the polynomials on the unit 
circle. The generating function (1.2) now takes the form

$$
\left(1-z e^{-i \theta}\right)^{-\alpha}\left(1-z e^{i \theta}\right)^{-\beta}=\sum_{n=0}^{\infty} C_{n}^{(\alpha, \beta)}\left(e^{i \theta}\right) z^{n}
$$

for $|z|<1$ and $\theta \in[0, \pi]$.

In what follows, we will show that the set of Heisenberg polynomials on the unit circle provides a simple non-trivial example in the uniform treatment of Darboux's method given in 18 .

\section{UNIFORM TREATMENT OF DARBOUX'S METHOD}

Darboux was the first to consider the asymptotic behavior of the coefficients $a_{n}$ in the Maclaurin expansion

$$
F(z)=\sum_{n=0}^{\infty} a_{n} z^{n}
$$

where $F(w)$ has only a finite number of singularities on its circle of convergence, all of which are algebraic in nature. A method was introduced to obtain the asymptotic expansion for $a_{n}$ as $n \rightarrow \infty$. The method, named after Darboux, indicates that the contribution to the expansion comes from the singularities on the circle of convergence; cf., e.g., Olver [12] and Wong [17] for the classical Darboux method.

If the singularities are allowed to move around on the circle of convergence, Darboux's method will continue to work provided that their essential configuration remains the same (although their relative positions vary). However, this method breaks down when two or more singularities coalesce with each other. A uniform treatment was later given by Fields [5], in which he considered generating functions of the type

$$
F(z, \theta)=(1-z)^{-\lambda}\left[\left(e^{i \theta}-z\right)\left(e^{-i \theta}-z\right)\right]^{-\Delta} f(z, \theta)=\sum_{n=0}^{\infty} a_{n}(\theta) z^{n}
$$

for $|z|<1$, where $\lambda$ and $\Delta$ are bounded quantities, the branches are chosen such that both $(1-z)^{-\lambda}$ and $\left[\left(e^{i \theta}-z\right)\left(e^{-i \theta}-z\right)\right]^{-\Delta}$ reduce to 1 at $z=0$, and $f(z, \theta)$ is analytic in $|z|<e^{\eta}$ with $\eta>0$ not depending on $\theta$. The results in [5] were regarded as being too complicated to apply; see, e.g., 4, p. 167].

The problem of uniform treatment was revisited by Wong and Zhao [18, in which they first addressed a special case of (2.2) with $\lambda=0$, namely

$$
F(z, \theta)=\left[\left(e^{i \theta}-z\right)\left(e^{-i \theta}-z\right)\right]^{-\alpha} f(z, \theta)=\sum_{n=0}^{\infty} a_{n}(\theta) z^{n} .
$$

Uniform asymptotic expansion was derived in terms of the Bessel functions $J_{\alpha \pm 1 / 2}(n \theta)$, valid for $\theta \in[0, \pi-\varepsilon], \varepsilon>0$. The general case considered in [18] deals with many coalescing singularities,

$$
F(z, \theta)=\left\{\prod_{k=1}^{q}\left(z_{k}(\theta)-z\right)^{-\alpha_{k}}\right\} f(z, \theta)=\sum_{n=0}^{\infty} a_{n}(\theta) z^{n},
$$

where $\left|z_{k}(\theta)\right|=1, q \geq 2, \alpha_{k}$ are constants, and $f(z, \theta)$ is the same function as in (2.2) and (2.3). In (2.4), all the branch points $z_{k}(\theta)$ approach 1 as $\theta$ tends to 0; more precisely, $z_{k}(\theta)=e^{i \theta s_{k}(\theta)}$ with $s_{k}(0)=-i z_{k}^{\prime}(0)$ not vanishing. A uniform 
asymptotic expansion for the general case was presented in [18] in terms of a set of special functions $T_{l}, l=1,2, \cdots, q$.

In a follow-up paper, Bai and Zhao [1] extended the essential ideas in [18] to study the classical Jacobi polynomials.

As mentioned in the last section and shown in (1.6), the set of Heisenberg polynomials serves as a good example of the uniform Darboux treatment, which is simple (only two singularities) and non-trivial (the exponents $\alpha$ and $\beta$ may be different). To derive the asymptotic expansion of $C_{n}^{(\alpha, \beta)}\left(e^{i \theta}\right)$, we apply Theorem 2 of [18]. To this end, we define

$$
T_{l}(x)=\frac{1}{2 \pi i} \int_{\Gamma_{0}} s^{l-1}(s-i)^{-\beta}(s+i)^{-\alpha} e^{x s} d s, l=1,2,
$$

where $\Gamma_{0}$ is a Hankel-type loop which starts and ends at $-\infty$ and encircles $s= \pm i$ in the positive sense; cf. [18, (4.7)]. We further introduce an auxiliary function

$$
h_{0}(s, \theta)=e^{i \theta(\alpha-\beta)}\left[\frac{e^{i \theta}-e^{-\theta s}}{(s+i) \theta}\right]^{-\alpha}\left[\frac{e^{-i \theta}-e^{-\theta s}}{(s-i) \theta}\right]^{-\beta}
$$

and a sequence of functions $\left\{h_{k}(s, \theta)\right\}_{k=0}^{\infty}$ defined inductively by

$h_{k}=\alpha_{k}(\theta)+s \beta_{k}(\theta)+\left(s^{2}+1\right) g_{k}(s, \theta), h_{k+1}=\frac{s^{2}+1}{\theta}\left\{\frac{\alpha-1}{s+i}+\frac{\beta-1}{s-i}-\frac{d}{d s}\right\} g_{k}(s, \theta)$

for $k=0,1, \cdots$. The coefficients $\alpha_{k}(\theta)$ and $\beta_{k}(\theta)$ are determined by requiring all $h_{k}(s, \theta)$ and $g_{k}(s, \theta)$ to be analytic in $\mathcal{D}=\left\{s: \operatorname{Re} s \geq-\frac{\eta}{\theta},|s \pm i|<\frac{2 \pi}{\theta}\right\}$. The uniform asymptotic expansion is given in the following theorem:

Theorem 1. For $\theta \in[0, \pi-\delta]$ with arbitrary $\delta>0$, we have

$$
C_{n}^{(\alpha, \beta)}\left(e^{i \theta}\right)=\theta^{1-\alpha-\beta} T_{1}(n \theta) \sum_{k=0}^{m-1} \frac{\alpha_{k}(\theta)}{n^{k}}+\theta^{1-\alpha-\beta} T_{2}(n \theta) \sum_{k=0}^{m-1} \frac{\beta_{k}(\theta)}{n^{k}}+\varepsilon_{\theta, m},
$$

where $\left|\alpha_{k}(\theta)\right| \leq M_{k},\left|\frac{\beta_{k}(\theta)}{\theta}\right| \leq M_{k}$, for $k=0,1,2, \cdots$, and

$$
\left|\varepsilon_{\theta, m}\right| \leq M_{m} \theta^{1-\alpha-\beta} n^{-m}\left\{\left|T_{1}(n \theta)\right|+\left|T_{2}(n \theta)\right|\right\},
$$

for $m=1,2, \cdots$. The positive constants $M_{k}, k=1,2, \cdots$, are independent of $\theta$ for $\theta \in[0, \pi-\delta]$. The coefficients $\alpha_{k}(\theta)$ and $\beta_{k}(\theta)$ are given iteratively in (2.7), with

$\alpha_{0}=\frac{e^{i \theta \alpha}}{2}\left(\frac{\sin \theta}{\theta}\right)^{-\alpha}+\frac{e^{-i \theta \beta}}{2}\left(\frac{\sin \theta}{\theta}\right)^{-\beta}, \beta_{0}=\frac{e^{i \theta \alpha}}{2 i}\left(\frac{\sin \theta}{\theta}\right)^{-\alpha}-\frac{e^{-i \theta \beta}}{2 i}\left(\frac{\sin \theta}{\theta}\right)^{-\beta}$.

\section{Special FunCtions $T_{1}$ And $T_{2}$}

By expanding the slowly varying factor in the integrand of (2.5) in a uniformly convergent power series of $1 / s$ and integrating term by term, we obtain

$$
T_{1}(x)=x^{\alpha+\beta-1} \sum_{k=0}^{\infty} \sum_{l=0}^{k} \frac{i^{k}(-1)^{l}(\alpha)_{l}(\beta)_{k-l}}{l !(k-l) ! \Gamma(\alpha+\beta+k)} x^{k},
$$

where $x^{\alpha+\beta-1}$ is positive for real positive $x$. It is easily seen that $x^{-\alpha-\beta+1} T_{1}(x)$ is an entire function. From (2.5), it is also readily verified that $T_{2}(x)=T_{1}^{\prime}(x)$ in the cut plane $x \in \mathbb{C} \backslash(-\infty, 0]$. 
The behavior of $T_{1}(x)$ at the origin is exhibited by the leading terms in (3.1). The asymptotic behavior of $T_{l}(x)$ as $x \rightarrow+\infty$ has already been given in [18, (4.45)]. Moreover, with $a=2-\alpha-\beta$ and $b=\beta-\alpha, T_{1}(x)$ satisfies the differential equation

$$
x T_{1}^{\prime \prime}+a T_{1}^{\prime}+(x-b i) T_{1}=0 ;
$$

cf. 18, (4.52)]. This equation is of Laplace type in the sense that the coefficients are linear in $x$. We proceed to show that $T_{1}(x)$ is connected to the confluent hypergeometric function via the following change of variables:

$$
T_{1}(x)=x^{1-a} e^{i x} y(x), z=-2 i x .
$$

Substituting (3.3) in (3.2) leads to the Kummer equation

$$
z \frac{d^{2} y}{d z^{2}}+[(2-a)-z] \frac{d y}{d z}-\frac{2-a-b}{2} y=0 .
$$

Taking into account the first two terms of the infinite series in (3.1), we have

$$
T_{1}(x)=\frac{1}{\Gamma(\alpha+\beta)} x^{\alpha+\beta-1} e^{i x} M(\alpha, \alpha+\beta,-2 i x),
$$

where $M$ is the Kummer function; cf. [13, p. 322]. (One may also derive this result directly from (2.5) and a Laplace inversion integral of this Kummer function [13, p. $327,(13.4 .13)]$.) Accordingly, from $T_{2}(x)=T_{1}^{\prime}(x)$ one has

$$
\begin{aligned}
T_{2}(x)= & \frac{(\alpha+\beta-1+i x) x^{\alpha+\beta-2} e^{i x}}{\Gamma(\alpha+\beta)} M(\alpha, \alpha+\beta,-2 i x) \\
& -\frac{2 i x^{\alpha+\beta-1} e^{i x}}{\Gamma(\alpha+\beta)} M^{\prime}(\alpha, \alpha+\beta,-2 i x),
\end{aligned}
$$

where $M^{\prime}(\gamma, \delta, z)=\frac{d}{d z} M(\gamma, \delta, z)$. Thus, substituting (3.5) and (3.6) in (2.8), we obtain an asymptotic expansion of the Heisenberg polynomials in terms of the Kummer function as follows:

Theorem 2. Assume that $\alpha$ and $\beta$ are real and fixed and that $z=\rho e^{i \theta}$ with $\rho>0$ and $\theta$ real. Then we have the compound asymptotic expansion ( $\$ 4.2$ of Chapter 4 in [12])

$$
\begin{aligned}
C_{n}^{(\alpha, \beta)}(z) \sim n^{\alpha+\beta-1} z^{n}\{M(\alpha, \alpha+\beta, & -2 i n \theta) \sum_{k=0}^{\infty} \frac{c_{k}(\theta)}{n^{k}} \\
& \left.+M^{\prime}(\alpha, \alpha+\beta,-2 i n \theta) \sum_{k=0}^{\infty} \frac{d_{k}(\theta)}{n^{k}}\right\}
\end{aligned}
$$

as $n \rightarrow \infty$, uniformly with respect to $\rho \in(0, \infty)$ and $\theta \in[0, \pi-\delta]$, where $\delta$ is an arbitrary constant such that $0<\delta \leq \pi$. Here the coefficients are given by

$$
c_{k}(\theta)=\frac{\alpha_{k}(\theta)+i \beta_{k}(\theta)}{\Gamma(\alpha+\beta)}+\frac{\beta_{k}(\theta) / \theta}{\Gamma(\alpha+\beta-1)}, d_{k}(\theta)=\frac{-2 i \beta_{k}(\theta)}{\Gamma(\alpha+\beta)}
$$

for $k=0,1,2, \cdots, \alpha_{k}(\theta)$ and $\beta_{k}(\theta)$ being defined as in Section 2. In particular, $c_{0}(\theta)=\frac{e^{i \theta \alpha}}{\Gamma(\alpha+\beta)}\left(\frac{\sin \theta}{\theta}\right)^{-\alpha}, d_{0}(\theta)=\frac{e^{-i \theta \beta}}{\Gamma(\alpha+\beta)}\left(\frac{\sin \theta}{\theta}\right)^{-\beta}-\frac{e^{i \theta \alpha}}{\Gamma(\alpha+\beta)}\left(\frac{\sin \theta}{\theta}\right)^{-\alpha}$. 
When the parameters $\alpha$ and $\beta$ in the above theorem are non-positive integers, the coefficients $c_{k}$ and $d_{k}$ in (3.7) all vanish; see (3.8). However, the asymptotic relation remains valid since the polynomials also vanish for large values of $n$; i.e., it is a trivial result.

For completeness, we write, by using (2.5) and (3.2), the asymptotic expansion in the special case $\alpha=\beta$; see also [18. In this case, we have

$$
T_{1}(x)=\frac{\sqrt{\pi}}{\Gamma(\alpha)}\left(\frac{x}{2}\right)^{\alpha-1 / 2} J_{\alpha-1 / 2}(x), T_{2}(x)=\frac{\sqrt{\pi}}{\Gamma(\alpha)}\left(\frac{x}{2}\right)^{\alpha-1 / 2} J_{\alpha-3 / 2}(x),
$$

where $J_{\nu}(x)$ is the Bessel function of the first kind; cf. [13, p. 217]. The expansion in Theorems 1 and 2 now takes the form:

Corollary 1. Assume that $\alpha$ is real and fixed and that $z=\rho e^{i \theta}$ with $\rho>0$ and $\theta$ real. Then we have the compound asymptotic expansion

$$
C_{n}^{(\alpha, \alpha)}(z) \sim \rho^{n} \frac{\sqrt{\pi}}{\Gamma(\alpha)}\left(\frac{n}{2 \theta}\right)^{\alpha-1 / 2}\left\{J_{\alpha-1 / 2}(n \theta) \sum_{k=0}^{\infty} \frac{\alpha_{k}(\theta)}{n^{k}}+J_{\alpha-3 / 2}(n \theta) \sum_{k=0}^{\infty} \frac{\beta_{k}(\theta)}{n^{k}}\right\}
$$

as $n \rightarrow \infty$, uniformly with respect to $\rho \in(0, \infty)$ and $\theta \in[0, \pi-\delta]$, where $\delta$ is an arbitrary constant such that $0<\delta \leq \pi$. Here $\alpha_{k}(\theta)$ and $\beta_{k}(\theta)$ are given in (2.7), and the leading coefficients are

$$
\alpha_{0}(\theta)=\cos (\alpha \theta)\left(\frac{\sin \theta}{\theta}\right)^{-\alpha} \text { and } \beta_{0}(\theta)=\sin (\alpha \theta)\left(\frac{\sin \theta}{\theta}\right)^{-\alpha} .
$$

\section{Asymptotic Zeros}

We complete this note by giving some remarks on the zeros of the Heisenberg polynomials. By (2.8), these zeros are approximated by the zeros of $T_{1}(n \theta)$. Thus, we shall later turn to consider the zeros of $T_{1}$. But, as a consistency check on our result, we first consider the special case $\alpha=\beta>-1 / 2$. In this case, the Heisenberg polynomials on the unit circle are related to the Gegenbauer polynomials via the identity

$$
C_{n}^{(\alpha, \alpha)}\left(e^{i \theta}\right)=C_{n}^{(\alpha)}(\cos \theta), \theta \in[0, \pi],
$$

which can be seen by comparing the generating functions in (1.6) and in [13, p. 448]. In this specific case, $C_{n}^{(\alpha, \alpha)}\left(e^{i \theta}\right)$ is known to have $n$ distinct zeros in the interval $0<\theta<\pi$.

The asymptotic behavior of the extreme zeros can be obtained from 3.10 and (3.11). Indeed, when $n \theta$ is bounded, the terms in the curly brackets in (3.10) can be written as $H:=\left(\alpha_{0}+\alpha_{1} / n\right) J_{\alpha-1 / 2}(n \theta)+\beta_{0} J_{\alpha-3 / 2}(n \theta)+O\left(n^{-2}\right)$; here, we have used the fact that $\beta_{k}(\theta)=O(\theta)=O(1 / n)$ for $k=0,1,2, \cdots$, as mentioned in Theorem 11. The quantity $H$ can be further expressed as

$$
H=\Omega\left[J_{\alpha-1 / 2}(n \theta)+\Delta J_{\alpha-1 / 2}^{\prime}(n \theta)+O\left(n^{-2}\right)\right]=\Omega\left[J_{\alpha-1 / 2}(n \theta+\Delta)+O\left(n^{-2}\right)\right],
$$

where $\Omega=\alpha_{0}+\left\{\alpha_{1}+\left(\alpha-\frac{1}{2}\right) \beta_{0} / \theta\right\} n^{-1}$ and $\Delta=\beta_{0} / \Omega$ both depend on $\theta$ and $n$. Note that $\Omega=\alpha_{0}+O(1 / n)=1+O(1 / n)$ and $\Delta=\left\{n \theta \Omega^{-1} \beta_{0} / \theta\right\} / n=O(1 / n)$ as 
$n \rightarrow \infty$. In (4.2), we have used the Taylor expansion of $J_{\alpha-1 / 2}(x)$ at $x=n \theta$ and the formula

$$
J_{\alpha-3 / 2}(x)=J_{\alpha-1 / 2}^{\prime}(x)+\frac{2 \alpha-1}{2 x} J_{\alpha-1 / 2}(x) ;
$$

cf. [13, p. 222]. Hence, $C_{n}^{(\alpha, \alpha)}(z)=0$ amounts to $H=0$, which implies

$$
J_{\alpha-1 / 2}(n \theta+\Delta)=O\left(n^{-2}\right) .
$$

From (3.11), it is readily seen that $\Delta=\alpha \theta+O\left(1 / n^{2}\right)$. Denoting by $j_{\alpha-1 / 2, k}$ the $k$ th positive zero of $J_{\alpha-1 / 2}(x)$, we have the asymptotic approximation

$$
\theta_{n, k}=\frac{j_{\alpha-1 / 2, k}}{n+\alpha}+O\left(n^{-3}\right)
$$

for the $k$ th zero of $C_{n}^{(\alpha, \alpha)}\left(e^{i \theta}\right)$ arranged in increasing order, where $k=1,2, \cdots$ are fixed and $\alpha>-1 / 2$. In deriving (4.3), we have made use of the fact that $J_{\alpha-1 / 2}^{\prime}$ does not vanish in a neighborhood of $j_{\alpha-1 / 2, k}$.

If we relax the restriction that $k$ be fixed while still requiring $\theta=O(1 / n)$ and $n \gg k \gg 1$, then formula (4.3) would continue to hold, with only a weaker error estimate. For instance, making use of the fact that

$$
j_{\alpha-1 / 2, k} \sim(k+(\alpha-1) / 2) \pi
$$

for $\alpha>1 / 2$ and $k \gg 1$ (cf. [13, p. 236, (10.21.19)]) and substituting this into (4.3) gives

$$
\theta_{n, k} \sim \frac{(\alpha-1) / 2+k}{n+\alpha} \pi
$$

for $\alpha>1 / 2$ and $n \gg k \gg 1$. The formula in (4.4) agrees with that in [14, p. 139, (6.6.3)] for $1 / 2<\alpha<1$.

On the other hand, taking the two leading terms in (3.10)-(3.11) and making use of the asymptotic approximation for the Bessel function [13, p. 223, (10.7.8)],

$$
J_{\nu}(x)=\sqrt{2 /(\pi x)} \cos (x-\nu \pi / 2-\pi / 4)+O\left(x^{-1}\right) \text { as } x \rightarrow+\infty,
$$

we have from (3.10)

$$
C_{n}^{(\alpha, \alpha)}(z)=\rho^{n} \frac{\theta^{-\alpha}}{\Gamma(\alpha)}\left(\frac{n}{2}\right)^{\alpha-1}\left(\frac{\sin \theta}{\theta}\right)^{-\alpha}\left\{\cos \left((n+\alpha) \theta-\frac{\alpha \pi}{2}\right)+O\left(\frac{1}{n \theta}\right)\right\}
$$

for $n \theta \gg 1$ and $\theta \in(0, \pi-\delta]$. It is readily seen that this asymptotic approximation agrees well with (4.4).

It is worth mentioning that if $\theta=\theta_{n, \nu}$ is a zero of $C_{n}^{(\alpha, \alpha)}\left(e^{i \theta}\right)$, then all points on the ray $\arg z=\theta_{n, \nu}$ are zeros of $C_{n}^{(\alpha, \alpha)}(z)$. This fact follows readily from the homogeneity of the Heisenberg polynomials, namely, $C_{n}^{(\alpha, \beta)}\left(\rho e^{i \theta}\right)=\rho^{n} C_{n}^{(\alpha, \beta)}\left(e^{i \theta}\right)$ for $\rho>0$ and $\theta \in(-\pi, \pi]$.

Now, we turn to the asymptotic zeros of the special function $T_{1}(x)$, whose asymptotic properties were investigated in [18. It is of interest to consider the zeros of $T_{1}(x)$ for $x>0$ when $\alpha \neq \beta$. Without loss of generality, we assume that $\beta>\alpha$.

Proposition 1. The function $T_{1}(x)$ possesses no positive zeros when $\alpha+\beta>0$. 
Proof. We use an argument similar to that given in Ince [11, p. 512]. Write $T_{1}(x)=x^{\frac{1}{2}(\alpha+\beta)-1} I(x)$. Equation (3.2) gives

$$
I^{\prime \prime}(x)+\left[g_{1}(x)+i g_{2}(x)\right] I(x)=0,
$$

where the functions

$$
g_{1}(x)=1-\frac{a(a-2)}{4 x^{2}} \text { and } g_{2}(x)=-\frac{b}{x}
$$

are real and $g_{2}(x)<0$ for $x>0$. From (4.7) we have

$$
\left(\bar{I} I^{\prime}\right)^{\prime}=\left|I^{\prime}\right|^{2}+\bar{I} I^{\prime \prime}=\left\{\left|I^{\prime}\right|^{2}-g_{1}|I|^{2}\right\}-i\left\{g_{2}(x)|I|^{2}\right\} .
$$

Integrating over an arbitrary interval $\left[x_{1}, x_{2}\right]$ and taking the imaginary part, one obtains

$$
\operatorname{Im}\left[\overline{I(x)} I^{\prime}(x)\right]_{x_{1}}^{x_{2}}+\int_{x_{1}}^{x_{2}} g_{2}(x)|I(x)|^{2} d x=0 .
$$

From (3.1), it is straightforward to verify that when $\alpha+\beta>0, \lim _{x \rightarrow 0^{+}} \operatorname{Im}\left[\overline{I(x)} I^{\prime}(x)\right]$ $=0$. Hence, for $x>0$,

$$
\operatorname{Im}\left[\overline{I(x)} I^{\prime}(x)\right]=-\int_{0}^{x} g_{2}(s)|I(s)|^{2} d s>0,
$$

from which it follows that $I(x)$, and consequently $T_{1}(x)$, has no zero in the interval $(0, \infty)$.

In view of Theorem 2 , we conclude that for large values of $n$ there are no zeros of $C_{n}^{(\alpha, \beta)}(z)$ in the complex $z$-plane when $\alpha \neq \beta$ and $\alpha+\beta>0$. In our discussion of asymptotic zeros of $C_{n}^{(\alpha, \beta)}(z)$, we have not addressed the cases when $\alpha=\beta \leq-1 / 2$ and when $\alpha \neq \beta$ with $\alpha+\beta \leq 0$.

\section{REFERENCES}

[1] X.-X. Bai and Y.-Q. Zhao, A uniform asymptotic expansion for Jacobi polynomials via uniform treatment of Darboux's method, J. Approx. Theory, 148(2007), 1-11. MR2356572 (2009c:33017)

[2] C. F. Dunkl, The Poisson kernel for Heisenberg polynomials on the disk, Math. Z., 187(1984), 527-547. MR760053 (85m:42027)

[3] C. F. Dunkl, Boundary value problems for harmonic functions on the Heisenberg group, Canad. J. Math., 38(1986), 478-512. MR833580 (87f:22006)

[4] A. Erdélyi, Uniform asymptotic expansion of integrals, 149-168, in Analytic Methods in Mathematical Physics, R. P. Gilbert, R. G. Newton, eds., Gordon and Breach, New York, 1970. MR0336181 (49:957)

[5] J. L. Fields, A uniform treatment of Darboux's method, Arch. Rational Mech. Anal., 27(1967), 289-305. MR0220984 (36:4036)

[6] G. B. Folland, A fundamental solution for a subelliptic operator, Bull. Amer. Math. Soc., 79(1973), 373-376. MR0315267 (47:3816)

[7] G. B. Folland and E. M. Stein, Estimates for the $\bar{\partial}_{b}$ complex and analysis on the Heisenberg group, Comm. Pure Appl. Math., 27(1974), 429-522. MR0367477(51:3719)

[8] G. Gasper, Orthogonality of certain functions with respect to complex-valued weights, Canad. J. Math., 33(1981), 1261-1270. MR638380 (83a:33014)

[9] P. C. Greiner, Spherical harmonics on the Heisenberg group, Canad. Math. Bull., 23(1980), 383-396. MR602590 (82e:43009)

[10] R. Howe, On the role of the Heisenberg group in harmonic analysis, Bull. Amer. Math. Soc., 3(1980), no. 2, 821-843. MR0578375 (81h:22010)

[11] E. L. Ince, Ordinary Differential Equations, Dover, New York, 1956. MR0010757(6:65f) 
[12] F. W. J. Olver, Asymptotics and Special Functions, Academic Press, New York, 1974. MR0435697(55:8655)

[13] F. W. J. Olver, D. W. Lozier, R. F. Boisvert and C. W. Clark, NIST Handbook of Mathematical Functions, Cambridge University Press, 2010. MR2723248(2012a:33001)

[14] G. Szegő, Orthogonal Polynomials, 4th ed., American Mathematical Society, Providence, Rhode Island, 1975. MR0372517 (51:8724)

[15] N. M. Temme, Uniform asymptotic expansion for a class of polynomials biorthogonal on the unit circle, Constr. Approx., 2(1986), 369-376. MR892162 (88e:42047)

[16] S. Thangavelu, Harmonic Analysis on the Heisenberg Group, Birkhäuser, Boston, 1998. MR:1633042 (99h:43001)

[17] R. Wong, Asymptotic Approximations of Integrals, Academic Press, Boston, 1989. MR.1016818 (90j:41061)

[18] R. Wong and Y.-Q. Zhao, On a uniform treatment of Darboux's method, Constr. Approx., 21(2005), 225-255. MR2107940(2005h:41064)

Department of Mathematics, ZhongShan University, GuangZhou 510275, People's Republic OF CHinA

Department of Mathematics, City University of Hong Kong, Tat Chee Avenue, KowLOON, HONG KONG

Department of Mathematics, ZhongShan University, GuangZhou 510275, People's RePublic OF CHINA

E-mail address: stszyq@mail.sysu.edu.cn 